\title{
STOP: THINK SERIES: An abnormal chest x-ray and "Asthma"'
}

\author{
Praveen Bhatia $^{\mathrm{a}, *}$, S. Karthik ${ }^{\mathrm{b}}$, J.F. O’Reilly ${ }^{\mathrm{c}}$ \\ ${ }^{a}$ North Manchester General Hospital, Respiratory Department, Manchester, UK \\ ${ }^{b}$ Chest Clinic, Blackpool Victoria Hospital, UK \\ c University Hospital Aintree, Liverpool, UK
}

Received 1 December 2003; accepted 13 May 2004

\section{Case history}

A 60 year old lady was referred to the chest clinic with a six-month history of cough and intermittent wheeze. Previously, she had been reasonably fit. She had no past medical history of eczema or cardiac illness. She did mention that as a child she had had some chest symptoms and was hospitalised once when she was a teenager. She was single, worked as a secretary in an office and had never smoked. She had consulted her doctor soon after her symptoms began, was diagnosed with asthma, and was started on asthma medication in a stepwise pattern. She was now on treatment with high-dose inhaled steroids, twice daily long acting beta 2 agonist, regular short acting beta 2 agonist, once daily montelukast, and had recently been started on regular theophyllines. She had monitored her peak expiratory flow rates (PEFR) but these were always ranging between $200-220 \mathrm{~L} / \mathrm{min}$. On further enquiry there was no history suggestive of postnasal drip, but there was a six-month history of intermittent epigastric discomfort. She had noticed that at night sometimes she developed a bitter taste in the mouth. Apart from being mildly overweight and having slight epigastric discomfort, examination was unremarkable.

Her lung function tests including FEV1, FVC, FEV1/FVC, lung volumes and transfer factor were

${ }^{*}$ Corresponding author.

E-mail address: naviapo@aol.com (P. Bhatia). normal. She had a chest $x$-ray (Figure 1) while in the clinic. What is the diagnosis and what measures will you take in managing this patient?

\section{Answer}

The chest $x$-ray shows what is probably a large hiatus hernia with a fluid level. Confirmation could be obtained by performing a barium swallow. Her symptoms can be explained by gastro-oesophageal reflux disease (GORD). GORD has been reported in $10-40 \%$ of patients with chronic cough. Cough may occur due to microaspiration of oesophageal contents "into the tarynx and tracheobronchial tree or local irritation of the cough receptors within the lower oesophagus. Regurgitation can also lead to Wheeze which can be wrongly diagnosed as asthma. Normally the lower oesophageal sphincter, which is a localised area of specialised smooth muscle, is the main barrier to reflux. Since this lies below the diaphragm, intra-abdominal pressure as well as the oblique entry of the oesophagus into the stomach normally prevents reflux. In normal subjects this sphincter relaxes during swallowing, but at other times there are spontaneous transient relaxations.

The hiatus hernia may be incarcerated, a finding that could well alter patient management. Upper gastrointestinal endoscopy may be useful in determining the presence of erosive oesophagitis. It is recognised that patients with negative endoscopy reflux disease (NERD) and supra-oesophageal manifestations of reflux are more resistant to medical 


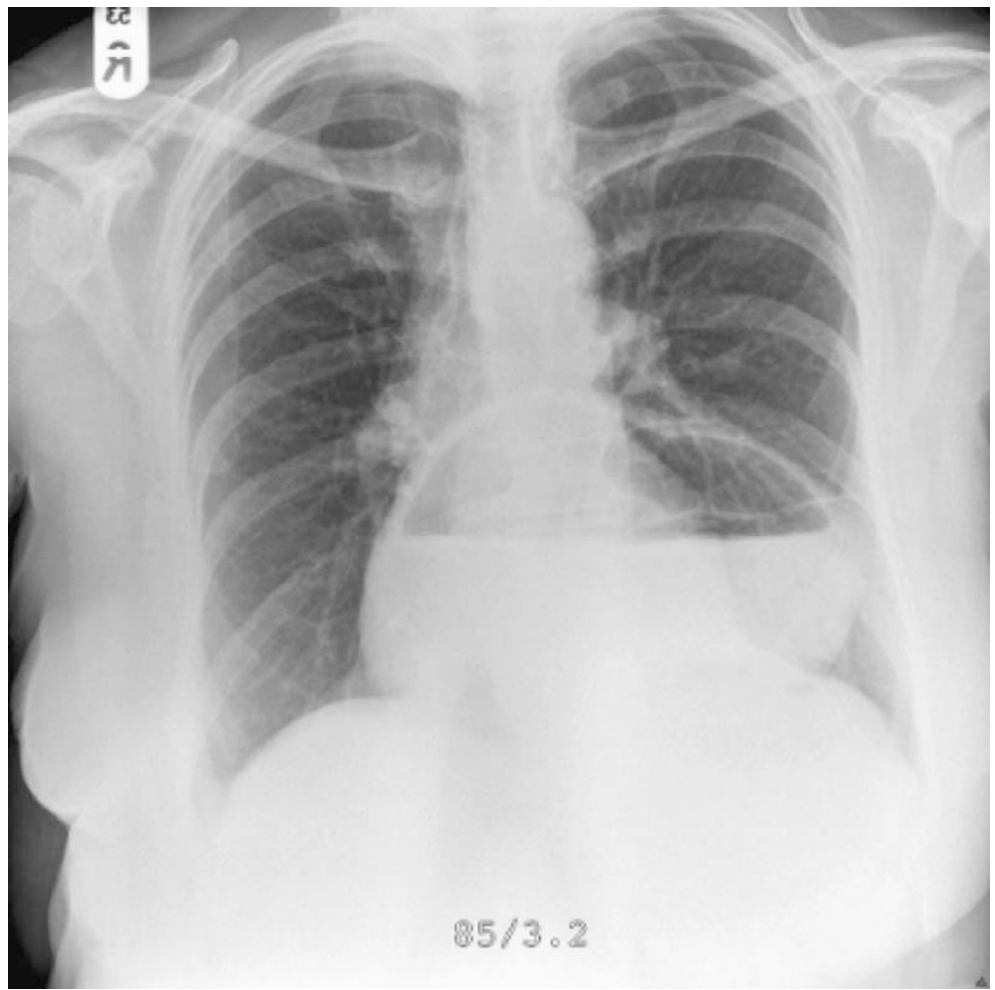

Figure 1

treatment with proton pump inhibitors (PPI) and surgery.

Dual probe 24-hour pH measurement may be useful in confirming acid reflux. It must be recognized that there is no "gold standard" diagnostic test for GORD. There are few, if any, indications today for one to consider doing the Bernstein test.

Provided there are no "alarm symptoms" or red flags (loss of weight, anaemia, dysphagia, odynophagia, gastrointestinal bleeding or recent onset of symptoms in a patient aged 45 or older), one could give a trial of therapy. A "step-down", approach is generally recommended, starting with the most potent class of drugi.e. PPI's. In some patients double dose PPI for 3 months may be re quired. However, in a recent cochrane review on the efficacy of conservative and surgical treatment of asthma in GORD, no overall improvement was found. They concluded that subgroups of patients may derive benefit, but that it appears difficult to predict responders. Patients with GORD not responding to adequate medical therapy often do not do well after anti-reflux surgery.

Finally, the value of lifestyle modification offers little benefit, being as effective as $\mathrm{H} 2$ receptor blocker therapy. Nonetheless, one should counsel the patient, especially regarding maintenance of ideal body weight, and sleeping with the head of the bed elevated. The latter is important in patients with large hiatus hernias as the hernia acts as a reservoir for reflux.

\section{Acknowledgements}

We are grateful for the assistance of $\mathrm{Dr}$ Herbie Schneider, Gastroenterologist, Johannesburg, South Africa, for his review of this paper.

Available online at www.sciencedirect.com

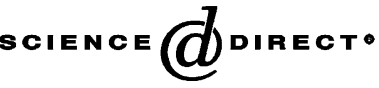

